\title{
Percepciones de los Estudiantes y Profesor Sobre el Uso de Aprendizaje Colaborativo Mediado por Computadores Inalámbricos ${ }^{1}$
}

\section{Perceptions of Students and Teacher on the Use of Collaborative Learning Supported by Wireless Computers}

\author{
Elsa Piedad Cabrera \\ Ministerio de Educación Nacional de Colombia
}

\begin{abstract}
El presente artículo describe las percepciones de los estudiantes de tercero medio y las de su profesor, en relación al uso de computadores portátiles inalámbricos conectados en red, como una herramienta mediadora del aprendizaje. Específicamente, las percepciones referidas a los logros que tanto estudiantes como profesor consideran obtienen con su uso así como aquellas referidas al funcionamiento del sistema. Se concluye aportando los efectos de dicha herramienta a nivel educativo en relación a los roles que deben desempeñar los actores dentro de la dinámica escolar, la importancia de clarificar los criterios de organización del grupo para que se produzca una interacción eficiente, así como también los aportes que desde los estudiantes se hace a la herramienta tecnológica.
\end{abstract}

Palabras Clave: aprendizaje colaborativo, computadores inalámbricos, percepciones, estudiantes y profesor.

\begin{abstract}
The following paper describes the perceptions of $11^{\text {th }}$ grade students and their teacher regarding the use of wireless portable computers connected in network, as a mediator tool of learning. Specifically, the perceptions referred to the achievements that students and teacher consider they obtain with their use, as well as those referred to the system's operation. The conclusions refer to the effects of this tool the education, regarding the roles that the actors should perform whit in the school dynamics, and the importance of clarifying the criteria for organizing the group, so that an efficient interaction takes place, as well as the students' contributions to the technological tool.
\end{abstract}

Keywords: collaborative learning, wireless computers, perceptions, students and teacher.

Diferentes aproximaciones desde la psicología han intentado dar respuesta sobre los procesos cognitivos que surgen cuando pequeños grupos trabajan colaborativamente haciendo uso del computador (CSCL - Computer Supported Collaborative Learning). Por la década de los 30, Vigotsky (1978) abre un nuevo camino en el estudio de los procesos cognitivos, donde el contexto socio-cultural en el que se encuentra el

Elsa Piedad Cabrera Murcia, Subdirección de Estándares y Evaluación, Ministerio de Educación Nacional de Colombia. La correspondencia relativa a este artículo deberá ser enviada a la autora al E-mail: epcabrer@puc.cl

1 Esta investigación se realizó gracias a la posibilidad que brindó el Proyecto Fondef DO1I1007 "Diseño, desarrollo e implementación de tecnología portable para el mejoramiento de la calidad de la educación" en el que trabajaban la Facultad de Ingeniería y la Escuela de Psicología de la Pontificia Universidad Católica de Chile.

Este trabajo es parte de los estudios preliminares de la tesis doctoral de la autora en el programa de Doctorado en Psicología, de la Escuela de Psicología de la Pontificia Universidad Católica de Chile.

La autora agradece al profesor Ricardo Rosas por su acompañamiento en la ejecución y revisión inicial de dicho artículo y a Mónica Correa, en el proceso de construcción en conjunto de los cuestionarios. individuo se considera clave en el desarrollo de dichos procesos. La importancia dada al contexto en Vigotsky (1978) trae aparejada la relevancia que tienen las herramientas como artefactos que median la interacción entre las personas y su contexto. Por tanto, las características psicológicas que constituyen al individuo se desarrollan en conexión con el sistema de acciones sociales y las actividades que se generan dentro del contexto en el que está inmerso (Minick, 1997).

$\mathrm{Al}$ usar las herramientas, en forma individual o grupal, éstas se constituyen en extensiones cognoscitivas que están a la base en el desarrollo de la cognición individual. A nivel grupal, los estados mentales propios pueden constituirse por los estados de los otros. Algunos diseños de programas y aplicaciones tecnológicas usadas en ambientes colaborativos, han sido concebidos como herramientas cognitivas. Esta consideración, estructura una situación de aprendizaje particular y favorece la emergencia o ampliación de las capacidades cognitivas en los individuos (Preiss \& Sternberg, 2006; Salomon, 1993; Vygotsky, 1978).

Lo anterior se ha evidenciado en estudios que han implementado el aprendizaje colaborativo como situación de aprendizaje mediada por tecnología fija, 
portátil e inalámbrica (ADP) en diferentes poblaciones y con diferentes tipos de tareas, con el propósito de identificar los beneficios sociales y cognitivos producidos. Por ejemplo, en ambientes CSCL con computador fijo se producen interacciones secuenciales más que simultáneas (Chiu, 2003), conflicto y vaguedad para dar solución a la tarea pues los participantes intentan tener el control de la misma fuente (p.e. el teclado, el mouse al tiempo) (Kynigos, 1999).

En grupos colaborativos mediados por computadores portátiles (laptop) se incrementa la interacción cara a cara y decrece el uso de herramientas tradicionales como la pizarra, cuadernos y libros de texto (Schaumburg, 2001). Sin embargo, en dichos ambientes se reporta la dificultad de generar un espacio colaborativo por la incompatibilidad de estilos -habilidades sociales y cognitivas-, las características del ambiente en el que se plantea la tarea (Haslam, 2000 citado en Lea, Rogers \& Postmes, 2002), así como los diferentes grados de motivación, los que originarían un trabajo individual más que grupal (Roschelle, Pea, Hoadley, Gordon \& Means, 2001).

El tipo de tecnología, las características particulares de la población y la estructura de la tarea, hacen que algunas aplicaciones tecnológicas sean más efectivas que otras dentro del contexto escolar (Roschelle et al., 2001; Zurita \& Nussbaum, 2004). Por ello, se ha dado paso a una nueva modalidad de aprendizaje colaborativo mediado por asistentes digitales personales (en adelante, CSCL con ADP). Pownell y Bailey (2000) argumentan que el uso de ADP dentro del aula va más allá de las ventajas que pueda tener en relación a su portabilidad, movilidad, etc., apuntando a destacar los beneficios obtenidos en el proceso de enseñanza-aprendizaje en profesores y estudiantes, el desarrollo del currículo, la dinámica al interior del grupo, así como la posibilidad de estudiar los procesos interaccionales producidos (Tatar, Roschelle, Vahey \& Penuel, 2003; Zurita \& Nussbaum, 2001; Zurita, Nussbaum \& Sharples, 2003).

Ciertamente, en un alto porcentaje las investigaciones realizadas en ambientes CSCL fijo, portátil o ADP han centrado su foco en los logros sociales y académicos alcanzados por los estudiantes y en los cambios producidos en la dinámica de enseñanza (Barros, Mizoguchi \& Verdejo, 2001; Danesh, Inkpen, Lau, Shu \& Booth, 2001; Gillies, 2003; Roschelle \& Pea, 2002; Shaumburg, 2001; Vahey \& Crawford, 2003; Veerman, 2000). En un porcentaje menor, se han centrado en los mecanismos sociales ocurridos al interior del grupo, las interacciones, las formas de colaboración dadas por las posiciones o roles tomados por los estudiantes (Barbieri \& Light, 1992; Hakkarainen \& Palonen, 2003; Inaba et al., 2001). Asimismo, se encontró poca información que permita contrastar estos resultados con la percepción de los mismos jóvenes y profesores, actores directamente involucrados en estas modalidades de aprendizaje (Jones, Connolly, Gear \& Read, 2001; Tatar et al., 2003; Yarnall, Penuel, Ravitz, Murray \& Means, 2001).

Tatar et al. (2003) realizaron una evaluación a gran escala durante un año, con profesores y estudiantes que hicieron uso de ADP. Este estudio explicitó que los profesores observaron un incremento en los aprendizajes de sus estudiantes, en las interacciones colaborativas y en la autonomía del estudiante. El uso inapropiado de información a la que pueden acceder los estudiantes y los problemas de funcionamiento de la tecnología, son algunas desventajas detectadas al usar dicha herramienta. Los estudiantes reportaron un incremento en la atención sobre la tarea, cuando trabajan con ADP. Dicho hallazgo es coincidente con los reportados por Yarnall et al. (2001) en su investigación realizada con grupos de $4^{\circ}$ y $5^{\circ}$ básico- en la que mostró cómo los estudiantes raramente se distraen de la tarea que desarrollan y que además, presentan una gran flexibilidad dentro del grupo en la forma como organizan su trabajo. Por otro lado, los profesores monitorean constantemente el trabajo de todos los grupos propiciándoles ayuda, intervenciones que son efectivas para el uso de la herramienta por parte de sus estudiantes. Tanto estudiantes como profesores confirman el importante rol que juegan estos últimos, pues su presencia aumenta la efectividad del trabajo en cada grupo.

Jones et al. (2001) evaluaron si los procesos que se dan en un grupo apoyados con ADP, un PC y un proyector, favorecen las experiencias de aprendizaje en la discusión y el debate, en 86 estudiantes universitarios. El profesor y los estudiantes coinciden en que el uso de dicha tecnología permite mayor focalización y atención en el tema que se discute, motivación, participación de los estudiantes y un manejo inmediato por parte del profesor de las confusiones o dificultades de los estudiantes. Estos por su parte, consideran que dicha tecnología les permite poder expresar sus ideas sin ser juzgados y desempeñar un rol más activo en su aprendizaje.

Evaluaciones realizadas por Palm Education Pioners (PEP) en Estados Unidos evidencian que un $90 \%$ de los profesores encuestados -que trabajan con ADP - afirman que su uso contribuye positivamente al aprendizaje de los estudiantes; un $84 \%$ de 
ellos concluye que usarla permitió mejorar la calidad de las actividades implementadas (Vahey \& Crawford, 2003). También se reporta que los ADP permiten complementar la actividad propuesta con el acceso que se puede tener a Internet desde un computador fijo, "los estudiantes pueden acceder a la web, bajar páginas relevantes a su ADP para futuros análisis" (Vahey \& Crawford, 2003,p. 8). Aunque esta característica se perfila como relevante para un trabajo más profundo, los profesores reportan que esta opción puede transformarse en un estímulo disruptivo (Tatar et al., 2003).

Considerando la valoración en el ámbito educativo de las bondades del CSCL con ADP así como de la poca información en relación a las percepciones de los actores sociales que participan de dicha dinámica, el presente estudio es llevado a cabo con el propósito de conocer las percepciones de los estudiantes y el profesor de enseñanza media de un liceo particular pagado, acerca del uso del ADP en forma colaborativa al desarrollar una actividad. Particularmente, se buscó con esta investigación dar respuesta a las siguientes preguntas:

1. ¿Cuál es el impacto que tiene en los estudiantes el trabajar en pequeños grupos haciendo uso de ADP en relación a su trabajo y a los logros que ellos consideran han alcanzado?

2. ¿Cuál es el rol que el profesor debería llevar a cabo para facilitar el manejo y el trabajo colaborativo en su salón de clase durante el transcurso de las actividades?

\section{Método}

\section{Participantes}

Los participantes fueron 30 estudiantes de tercero medio y su profesor de física, de un colegio ubicado en la Región Metropolitana de Santiago de Chile, perteneciente a un nivel socioeconómico medio-alto ${ }^{2}$. Se conformaron aleatoriamente 10 grupos de tres estudiantes utilizando el ADP como instrumento mediador en el aprendizaje colaborativo de contenidos de física. El profesor del área planificó cada una de las actividades planteadas durante el tiempo de observación, aspecto relevante en la dinámica escolar que se establece. Los participantes tienen una experiencia limitada en el trabajo colaborativo haciendo uso de dicha tecnología.

\section{Tarea}

Consistió en dar solución grupalmente a preguntas de selección múltiple que planteaban problemas de física rela-

2 El colegio hace parte de una muestra más amplia perteneciente al Proyecto de Investigación Fondef DO1I1007 enunciado anteriormente. cionados con el tema de "los fluidos". Para dar solución a ésta, los grupos de estudiantes podían considerar la información trabajada con anterioridad y/o hacer uso -si así lo requerían- de una guía con información pertinente al tema. El sistema les daba feedback de si su proceso de análisis y consecuente elección era o no el correcto, pudiendo avanzar si todos elegían la misma opción y si la retroalimentación recibida les indicaba que la respuesta era correcta.

\section{Tecnología Usada}

Se usó un dispositivo portátil (ADP) que utiliza una versión reducida del sistema operativo Microsoft Windows. Se trabajó con una plataforma PocketPCs que contiene un programa capaz de generar una actividad interactiva y colaborativa, que además de permitir su fácil portabilidad incorpora una red inalámbrica (Wi-Fi), que facilita la conexión inalámbrica de los ADP que se están usando en la sala de clase. El sistema cuenta con dos interfases. La primera permite al profesor ingresar a todos los estudiantes con un código, agruparlos azarosamente $\mathrm{y}$, distribuirles las actividades en las que trabajarán -en este caso- colaborativamente. En la segunda, que los estudiantes reciban dichas actividades y puedan dar inicio a la sesión colaborativa ${ }^{3}$ (Rosas et al., 2003).

\section{Instrumentos}

Para llevar a cabo este estudio exploratorio y recabar información sobre las percepciones de los estudiantes y el profesor de este nivel escolar se usó:

1. Grupos focales: Definida como la técnica más apropiada para recoger las percepciones de los actores sociales directamente involucrados en dicha dinámica escolar. Se realizaron dos grupos focales a partir de los cuales se intentó profundizar en las creencias y experiencias de los estudiantes al trabajar con los ADP. Estos estaban conformados por 6 estudiantes, 3 de rendimiento alto y 3 de medio-bajo según su desempeño en el área. Los roles que se desempeñan y el trabajo que se da al interior del grupo, los logros, las ventajas y desventajas del uso de ADP se constituyeron en los aspectos clave que se consideraron podrían generar discusión.

2. Pauta de Observación de Clase y del Profesor: Esta pauta recogió información en relación a la dinámica generada en los grupos de estudiantes, el rol del profesor y sus propias percepciones en relación a su rol y al clima del aula. Esta pauta se complementó con las respuestas dadas por el profesor a una entrevista semi-estructurada y a un cuestionario de 24 preguntas. Al igual que en los estudiantes, este cuestionario consta de dos partes. La primera de ellas apunta a identificar en el profesor cuál cree él, es la finalidad de usar ADP dentro del aula y, la segunda, el alcance que tiene su uso en el estudiante.

3. Cuestionario de Percepciones del Estudiante con relación al trabajo con ADP: Se aplicó un cuestionario después de terminado el trabajo colaborativo. Este constaba de 22 preguntas tendientes a recoger las percepciones que los estudiantes tienen del uso de los ADP como instrumento mediador de su aprendizaje. En las primeras 11

3 Este modelo está disponible al público en la página web http://www.mobilelearning.cl/ 
preguntas el estudiante marcaba la opción que mejor representara su pensamiento en relación a si clases organizadas colaborativamente con ADP o clases tradicionales les permitía alcanzar más, iguales o menores logros. La segunda, intentaba medir a partir de pares de afirmaciones las ventajas y desventajas del uso de CSCL con ADP dentro del aula. Tres jueces expertos evaluaron la pertinencia de los elementos que componían el cuestionario y además, realizaron sugerencias en la redacción.

\section{Procedimiento}

Inicialmente se explicitó a los estudiantes el objetivo de este estudio y el carácter voluntario de su participación. Se les explicó que se observaría durante cuatro semanas consecutivas, un día a la semana de hora y media de actividad, su trabajo colaborativo en un mismo grupo para dar respuesta a preguntas de selección múltiple referidas al tema de los fluidos. La herramienta tecnológica usada (ADP) permitía que los estudiantes estuvieran conectados en red y por tanto, pudiesen trabajar en la misma pregunta al mismo tiempo. Esta les proporcionaba a los estudiantes un feedback correcto o incorrecto o pónganse de acuerdo, que les indicaba cómo debían proceder en el desarrollo de su trabajo.

Durante este tiempo se fue registrando el clima generado al trabajar con dicha herramienta, así como el rol que el profesor desempeñaba en la clase y en cada uno de los grupos colaborativos. Esta decisión se sustenta en poder recoger información fidedigna de un ambiente natural como es el aula, sin afectar la dinámica que se produce en su interior. Una vez terminadas las observaciones en la quinta semana de clase, se pidió a los estudiantes y al profesor diligenciar el cuestionario individualmente sobre su percepción del trabajo con ADP durante 30 minutos. En esta misma semana se realizaron dos grupos focales, de 6 estudiantes cada uno (3 de nivel de rendimiento bajo y 3 de alto) con una duración de 45 minutos. Se eligió un espacio del colegio en el que los estudiantes se sintieran cómodos para expresar sus opiniones en relación a este trabajo.

Finalmente los datos recogidos con los cuestionarios fueron analizados en forma descriptiva con el propósito de identificar la percepción de los estudiantes y el profesor, referido a las diferencias que ellos consideran se dan con este tipo de dinámica frente a las clases tradicionales. Dicha información fue complementada con las observaciones realizadas al interior del aula. La información obtenida en los grupos focales fue analizada cualitativamente a partir de la codificación abierta del discurso emitido por los estudiantes. Dos jueces, extrajeron categorías que caracterizaron el discurso desde un nivel cercano a un nivel más abstracto del mismo. Con dichos análisis se pretende caracterizar las percepciones de los estudiantes y profesor sobre el uso de ADP en forma colaborativa dentro del aula, sin hacer ningún tipo de generalización.

\section{Resultados}

\section{Percepción de los Estudiantes Sobre el Uso de ADP en Clase}

Una primera ronda de codificación abierta del discurso de los estudiantes en los grupos focales, permitió identificar 77 categorías conceptuales. En una segunda, éstas se organizaron en cuatro categorías más abstractas a partir de las similitudes conceptuales que existían entre sí. Esto permitió reconstruir la percepción de los estudiantes en relación a esta nueva manera de aprender.

\section{Categoría 1: Del Sistema}

Los estudiantes consideran que el ADP como herramienta mediadora del aprendizaje tiene sus ventajas y desventajas en cuanto a su funcionamiento y su aporte al contexto del aula. Las ventajas sobre el funcionamiento hacen referencia a la estructura de la tarea, a la posibilidad de buscar la respuesta en conjunto y no su imposición: “... lo que tiene de bueno este método o sea la máquina, es que siempre una respuesta va a estar bien, siempre se va a saber la verdadera respuesta..." (FI/33), “... hay una respuesta inmediata, no como las pruebas...” (FI/34), “... lo que tienen de ventajoso los ADP es que tienen la información ahi, puedes estar haciendo un ejercicio usando la información, comparándola con el grupo y con el material que teni..." (FI/67). Para ellos, esta tecnología les da la posibilidad de desempeñarse individual y grupalmente haciendo el trabajo más equitativo: “... yo lo veo pa' gente que generalmente no se copia, como que generalmente se abusa o... se usa al que sabe más, entonces como que eso está bueno porque primero hay un trabajo individual y después se pone uno de acuerdo grupalmente..." (FI/22).

En cuanto a las desventajas los estudiantes consideran que el sistema ADP es mecánico y con restricciones en cuanto a la posibilidad de revisar el trabajo ya realizado: “... no es dinámico, es muy cuadrado, no tienen ningún incentivo... a menos de que te interese mucho [refiriéndose a la física]...” (FII/32), “... que por favor se pueda retroceder, porque muy complicado saber, cuando uno tiene una práctica de qué es más rápido para uno..." (FI/89). Consideran que las fallas tecnológicas presentadas es un factor desmotivante cuando el trabajo está en marcha: “... que se caiga el sistema es una lata... que se repitan las preguntas también es una falla, pues por ejemplo yo me acordaba de todas las respuestas sólo mirando el dibujito..." (FII/54).

En relación al aporte del trabajo con ADP al aula, la gran mayoría de los estudiantes consideran que su inclusión genera un espacio motivador y dinámi- 
co para el aprendizaje de la física: “... igual como entretenido [refiriéndose al trabajo con las ADP], porque tienen ilustraciones, igual como que entiendo más, como que se entretiene uno más..."(FII/ 4). También afirman que su uso trae como consecuencia positiva el aprovechamiento de la hora-clase y en general, el tiempo que se invierte en ella para realizar algunas actividades: “... como en la física uno se demora harto rato escribiendo la pregunta, haciendo los esquemas entonces es una manera de ahorrar tiempo... hacer una clase de hora y media mucho más productiva..." (FI/54).

En general los estudiantes piensan que el sistema podría ser mejor si existiera:

1. Un acompañamiento constante por parte del profesor u otra persona experta en el área en cada uno de los grupos: “... la idea de formar grupos, de trabajar con personas que no conocemos, yo creo que es ideal y... pero falta control, falta incentivo, no sé... el profesor debería acercarse a todos los grupos, debería ser todo un mismo grupo...” (FII/16), “... lo que pasa es que nunca vi al profesor cuando trabajé con las ADP asi que..." (FII/43), "de repente si uno tiene una pregunta hace 20 minutos y el profesor no llega, pues entonces filo y uno pasa a la siguiente, debería haber no sé, control... no sé un monitor estudiante de la U, por ejemplo..." (FII/50).

2. Antes de su uso una introducción al tema, para que la herramienta sea mediadora en el aprendizaje en forma efectiva: “... yo creo que el sistema puede ser mucho mejor si es que antes de usar las maquinitas viéramos el tema, viéramos muy por encima el tema y después trabajar... porque por lo menos partimos de una base... porque partimos sin nada...” (FII/45), “... es como hacer un ejercicio y ya, como que además debe ser complementado yo creo... está bien no tener siempre clases teóricas pero tampoco encuentro que sea muy bueno hacer puros ejercicios, con puras maquinitas..." (FI/10).

A juicio de los estudiantes el sistema mejoraría si se hace que sea más interactivo como se refleja con las siguientes intervenciones:

E1: "las respuestas, podrían haber más alternativas"

E2: "claro que podría ser que si no le achuntas ni a la primera ni a la segunda, no sé después de esa pregunta algo que te explique por qué te equivocaste ... o sea que te explique por qué es mayor por ejemplo... o por qué es esa respuesta...” (FII/52)

\section{Categoría 2: Trabajo en Grupo}

El cumplimento de ciertas condiciones en la organización del espacio físico y la conformación del grupo favorecen el trabajo en grupo con ADP. Una disposición del aula que facilite la interacción cara a cara son considerados necesarios para que se de un trabajo grupal eficiente. El siguiente diálogo entre dos estudiantes, ejemplifica dicha afirmación:

E1: "... me tocó en un grupo donde no me dejaban mucho participar..."

E2: “... y ¿por qué no podias participar?...”

E1: “... porque si te dan la espalda es medio dificil, como...[ejemplifica con el nombre de un compañero con el que trabajó alguna de las veces]"

E2: “... sí es dificil...” (FI/23-24-25)

La conformación del grupo es considerado un aspecto relevante para alcanzar buenos resultados, específicamente dos criterios. El primero referido al tamaño del grupo. Grupos extensos tornan difícil la dinámica de grupo. En sus palabras: “... cuando los grupos son muy grandes como que no funciona... yo trabajé en un grupo donde las personas trabajaban y alguien... supongo que es la a [imponiendo una respuesta al grupo], pero trabajé con otras personas... éramos tres en ese grupo y ahí funcionó muy bien..." (FI/23). El segundo, a los criterios usados para conformar los grupos a saber:

1. Por proximidad en espacio físico, “... los que están más cerca...” (FI/14), por lazos de empatía o amistad, “... qué bueno que pueda ser con la gente que la paso bien o más mis amigos...” (FI/54).

2. Por intereses en el área "... no sé poh yo creo que lo mejor es que al principio de la clase es ver quiénes tienen ganas de trabajar bien cachai..." (FI/18).

3. Por buen rendimiento académico. Los estudiantes con buen rendimiento, afirmaron que si están con compañeros de sus mismas condiciones académicas o no, pueden aprender más y ayudar a que sus otros compañeros aprendan, pero que a su vez pueden verse desfavorecidos cuando los estudiantes de nivel bajo no tienen disposición para aprender: “... también cuando uno trabaja con los que les va remal es bueno porque yo les puedo explicar y al mismo tiempo yo entender mejor y ayudarlos, pero también como les va mal les da lata, entonces... como todo hacerlo lo más rápido posible, es como el achunte para terminar antes cachai..." (FI/18).

Los de rendimiento bajo consideran que el trabajar con compañeros de nivel alto les favorece 
en su aprendizaje: “... si estás en el grupo de los que saben uno obviamente aprende más, pero si no es como que da lata...” (FII/22), “... si me toca con un grupo que le interesa el trabajo voy a tratar de seguirle el ritmo, en cambio si no es esto pues copio la respuesta..." (FII/33), pero también consideran que esta forma de trabajo les demanda un mayor esfuerzo y concentración, y que por tanto, prefieren una dinámica más tradicional “... no me gusta, no sé tal vez porque me toca hacer más esfuerzo, como que me gusta la discusión pero yo prefiero el profesor adelante y que me explique...” (FII/8), “... siento que hago un mayor esfuerzo con las ADP para no tener que hacer todo al achunte..." (FII/ 38).

4. Por niveles de conocimiento como se explícita en la siguiente discusión:

E1: "el punto es que si los formai tú igual no funciona, siempre van a estar unos que saben más y otros menos..."

E2: "pero hay una lista de las personas que saben más, si poni a una persona fuerte en cada grupo como que queda mejor formado".

E3: "hay que clasificar por niveles para poderlos agrupar... para que quede el que sabe más con el que sabe menos... un grupo parejo... por nivel de interés o por si saben o no..."

E4: "no porque si ponen gente que no le interesa no van a hacer nada..." (FII/23-25)

Según los estudiantes, las habilidades que el trabajo en grupo con ADP les demanda para el desarrollo eficiente de la tarea es un aspecto a considerar. Éstas guardan relación con la posibilidad de estar mayormente en actividad y concentrados en la tarea “... acá estamos un poco más activos, en realidad antes sólo nos explicaban y hacíamos preguntas en cambio aqui es completamente activo y que te pone a pensar...” (FI/8), “... siento que hago un esfuerzo mayor con las ADP para no tener que hacer todo al achunte..." (FII/38), "tienes que estar pendiente todo el tiempo de lo que estás haciendo para terminar todos los ejercicios...” (FII/41), “... siempre uno tiene que estar muy activo y poner atención y tratar de agarrar, porque la información te la están dando siempre..." (FI/36).

Asimismo consideran que el trabajo con ADP es positivo si el grupo logra trabajar en conjunto, de lo contrario el trabajo se vuelve poco colaborativo: “... bueno si el otro no se la jugai da lo mismo eso de 'pónganse de acuerdo' si el otro no se la jugai da lo mismo, porque se va uno al achunte, en otros grupos uno puede estar buscando en la guía, vamos pensando... [haciendo referencia al trabajo en equipo]” (FII/34), “... es tener como el sentido de grupo...” (FI/78), "en algunos grupos uno puede aportar más o aprender más y en otros grupos que se dice poner esta opción y sino es poner esta otra...” (FI/81), “... he tenido experiencias en donde la he pasado re mal cachai... y después me da lata trabajar así, porque cuando encuentro a alguien que le importa más o menos igual que a mí bien cachai pero al que no... " (FI/85).

\section{Categoría 3: Logros}

Se reportan beneficios a nivel social y cognitivo. Los beneficios sociales guardan relación con la posibilidad que ellos tienen de: (a) entender y conocer al otro, “... la idea de formar grupos, de trabajar con personas que no conocemos, yo creo que es ideal..." (FII/16); (b) actitudes de colaboración hacia el otro: “... el hecho de hacer los grupos, hace que en conjunto se trabaje para seguir avanzando..." (FII/2), “... yo creo que en el minuto que tenga un grupo que sabe menos igual hay que enseñar...” ( FI/76), “... y sino entiendes que no te digan es la $a . .$. [hace referencia a la elección de opciones] sino cómo se hace, es también como tener el sentido como de grupo... no sirve de nada que te digan cuál es o pon ésta porque ésta es la buena..." (FI/ 78).

Para los estudiantes los beneficios cognitivos que les reporta esta modalidad se reflejan en entender y aprender más de la materia, así como hacer uso de sus habilidades comunicativas. Algunos opinan que: “... siempre hemos discutido, incluso cuando todos hemos estado de acuerdo igual, como que nos complementamos la información, eh... como que se basa mucho en dialogar..." (FI/19), "encuentro que es entretenido discutir la materia..." (FI/60), “... yo les puedo explicar y al mismo tiempo o entender mejor y ayudarlos..." (FI/18).

En general los estudiantes piensan que el profesor hace uso de esta modalidad de trabajo para:

1. Desarrollar en ellos habilidades sociales y cognitivas. Opinan que esta forma es usada “... para que discutamos, que aprendamos juntos” (FII/42), “... fortalecer el trabajo en grupo, discutir y aprender de nuestros similares..." (FI/ 51), “... desarrollar el razonamiento...” (FI/52).

2. Cambiar la dinámica que se ha dado dentro del aula tradicionalmente "... estar trabajando todo el rato, porque en las otras formas de clases no 
estamos trabajando todo el rato..." (FI/53), "llevar a cabo un trabajo más didáctico, que aprendamos todos juntos y a la par como para cambiar el sistema" (FI/42).

\section{Categoría 4: Roles}

Los estudiantes reconocen que el trabajo en pequeños grupos con ADP es eficiente si se aprende recíprocamente, es decir, se enseña y se aprende en conjunto con los otros compañeros. Consideran que la clave para ello es aprender a enseñar y a recibir la información, de lo contrario el trabajo en equipo se torna dispendioso. En sus palabras: “... es real que hay gente que sabe más y otra que sabe menos, hay gente que... como que tenis que... por hacer el grupo, enseñar y recibir información grupal...” (FI/75), “... es necesario un compromiso de todos y ver la decisión de yo te enseño o a ver enséñame, porque igual es pura información cachai..."(FI/77).

Percepción de Logro de los Estudiantes en Relación con la Metodología de CSCL con ADP

Los anteriores resultados fueron enriquecidos con los obtenidos a partir del cuestionario aplicado a estudiantes sobre sus percepciones en relación al trabajo con ADP.

Sobre una base descriptiva (ver Tabla 1) puede afirmarse que los estudiantes perciben que a diferencia de las clases tradicionales, las colaborativas con ADP favorecen una mayor concentración en la tarea, participación y aprovechamiento del tiempo en clase, apoyo entre sí, actitudes positivas para escuchar a sus compañeros y oportunidad para explicar a los otros formas de dar desarrollo a la tarea.

Asimismo, un 54.8\% de los estudiantes considera que la atención recibida por parte del profesor se ve disminuida en este tipo de dinámica, mientras que un $35.5 \%$ considera que recibe la misma atención que cuando se está en clase tradicional. De igual manera, los estudiantes perciben que dicha herramienta les brinda la misma posibilidad que cuando trabajan en dinámicas tradicionales de resolver dudas, entender, aprender e interesarse por la materia.

\section{Percepción del Profesor en Relación al Trabajo con ADP}

Aunque sólo dispongamos de información proporcionada por un profesor - una entrevista semiestructurada y un cuestionario-, es interesante poder analizar en forma cualitativa su percepción en relación al uso de las ADP en clase. A continuación se presentan en conjunto los resultados de ambos instrumentos.

El profesor valoró el uso de ADP como una herramienta que permite el desarrollo de habilidades cognitivas y sociales en los estudiantes. Esta favorece un rol más activo del estudiante en clase, trabajar cooperativamente, entender, repasar, complementar los conocimientos en el área y alcanzar logros académicos. Asimismo, considera que la posibilidad de participar y aprender activamente en grupo está dada por la dinámica que se establece y la manera en que está estructurado el sistema: “... la posibilidad de aprender, en base a lo que en papel o pizarra sería un cuestionario o ejercicios, pero con la posibilidad de tener una respuesta inmediata..." (E1/5).

Tabla 1

Porcentajes que caracterizan la percepción de los estudiantes de los logros y sin tecnología

\begin{tabular}{lccc}
\hline Indicador que compara el trabajo con y sin ADP & Más & Igual & Menor \\
\hline Entender la materia & $35.5 \%$ & $41.9 \%$ & $22.6 \%$ \\
Concentrarse en la tarea & $61.3 \%$ & $25.8 \%$ & $12.9 \%$ \\
Aprender la materia & $32.3 \%$ & $41.9 \%$ & $25.8 \%$ \\
Participar en el grupo & $74.2 \%$ & $22.6 \%$ & $3.2 \%$ \\
Intereses por la actividades & $35.5 \%$ & $48.4 \%$ & $16.1 \%$ \\
Aprovechamiento del tiempo & $41.9 \%$ & $38.7 \%$ & $19.4 \%$ \\
Apoyar a mis compañeros & $71.0 \%$ & $29 \%$ & $0 \%$ \\
Escuchar a mis compañeros & $51.6 \%$ & $38.7 \%$ & $9.7 \%$ \\
Oportunidad para explicar & $64.5 \%$ & $32.3 \%$ & $3.2 \%$ \\
Despejar dudas & $22.6 \%$ & $45.2 \%$ & $32.3 \%$ \\
Recibir atención del profesor & $9.7 \%$ & $35.5 \%$ & $54.8 \%$ \\
\hline
\end{tabular}


Por otro lado, el profesor considera que el uso rutinario del ADP puede desfavorecer el proceso de enseñanza-aprendizaje. En sus palabras "En una primera etapa resulta bastante motivante y creo que la razón principal se explica por la novedad que significan la Palm [ADP] y el estilo de trabajo. Una vez que se entra en la rutina esta motivación se reduce" (E1/8). Él rescata el uso de dicha herramienta como un recurso que media el aprendizaje, un elemento que complementa el desarrollo de la actividad y no es el centro de la misma: “... creo que las Palm [ADP] son un recurso metodológico valioso; pero, igual que cualquier otro, debe constituir un complemento del trabajo pedagógico. En otras palabras, no puede ser el centro único en torno al cual gire las clases, así como tampoco deben serlo la pizarra, el laboratorio o la multimedia" (E1/8).

El uso del ADP permite a los estudiantes la interacción con sus pares para resolver dudas: “... El alumno tiene ante sí una pregunta y opciones de respuesta. Al marcar una respuesta es muy probable que aclare su duda. Si se equivocó, la aclarará en la discusión con sus compañeros..." (E1/13), incrementar el aprovechamiento del tiempo de la clase: “... creo que permite avanzar bastante más rápido que con otros métodos, junto con la ventaja de cubrir todos los contenidos. No cabe, por ejemplo la posibilidad de que al profesor se le olvide tratar tal o cual contenido" (E1/12), y la posibilidad de aprender y prepararse mejor para las pruebas de ingreso a la universidad, en especial a aquellos estudiantes de rendimiento promedio.

La percepción de su rol dentro de la dinámica colaborativa es otro aspecto importante que aparece en el discurso del profesor. Su rol se reparte entre el adecuado funcionamiento del sistema y el desempeño de sus estudiantes. La primera evaluada por el profesor como una función evidente y necesaria para que funcione el sistema. La segunda, como su rol principal cuando se lleva a cabo el trabajo en grupos colaborativos con ADP. Éste se centra en la supervisión y monitoreo del trabajo del estudiante y algunas funciones relacionadas con mantener un adecuado clima de aula. En sus palabras los roles que desempeñan son “... bastante evidentes. Por ejemplo hacer funcionar las ADP, seleccionar y enviar las actividades adecuadas, recoger la información, etc. Entre los roles propiamente pedagógicos son importantes la etapa de motivación inicial, la atención de consultas y aclaración de dudas que surjan en cada grupo..." (E1/6).
Asimismo, argumenta que su rol es el mismo en las clases con y sin ADP ya que en ambas debe prestar la misma cantidad de atención al desempeño de sus estudiantes: "Las necesidades de atención deben ser altas en ambas modalidades, es la única manera de aprender los contenidos" (E1/9). El aprendizaje con ADP presenta ventajas en el estudiante y desventajas en su rol. A la par de rescatar en los estudiantes la posibilidad que tienen de hacerse responsables de su propio proceso de aprendizaje considera que esto trae consigo una pérdida de control de su trabajo en el aula: “... en el trabajo con la ADP la instancia de concentrarse en alguna idea o concepto es responsabilidad del alumno... pero también origina en gran medida que el profesor pierda el control de lo que está ocurriendo..." (E1/9).

\section{Discusión}

En este artículo hemos descrito las percepciones que los estudiantes y el profesor de tercero medio, tienen cuando se trabaja CSCL con ADP en clase de física. Los resultados de este estudio permiten concluir desde el campo educativo y psicológico, así como desde el tecnológico (ADP).

Respecto de los efectos educativos generados por usar ADP, es posible identificar su impacto en la percepción de los roles que deben desempeñar los actores involucrados. Para los estudiantes es relevante el rol que debe desempeñar el profesor como mediador en el aprendizaje. Un rol que le permite ejercer mayor control y supervisión sobre la actividad y no solamente sobre ellos. Desde el profesor, su rol se centra más en el control y monitoreo de las acciones de cada estudiante. En este entendido, si el foco de atención del profesor está centrado solamente en el estudiante y no en la actividad, esto imposibilita el desarrollo de una dinámica más flexible, motivante e interactiva y se transforma en una actividad rutinaria y de simple ejercitación. Desde los estudiantes, su rol pasivo o parcial en el desarrollo de la clase es dejado de lado transformándose en agentes activos de su proceso de aprendizaje. El profesor pasa de ser un simple "inspector o supervisor" del trabajo de sus estudiantes a un rol más activo e interdependiente con la actividad misma.

El centrarse en la actividad por parte de los estudiantes como del profesor, es un elemento a considerar cuando se implementa el CSCL con ADP al interior del aula. Dicho resultado es consistente con la teoría de la actividad (Gifford \& Enyedy, 1999) que 
como marco metodológico del CSCL con ADP permite entender la actividad que se genera en esta forma de trabajo, como interdependiente de los otros elementos que se requieren para que exista actividad. Tanto la comunidad (los otros) como las herramientas (computador) son fuentes que el estudiante (agente) coordina durante el desarrollo de la actividad (objetivo) (Nardi, 1996 citado en Roschelle \& Pea, 2002). Centrarse por tanto en la actividad, conjuga un cambio de roles en los actores y en nuestro estudio se evidencia en el rol del profesor. Un rol de facilitador que genera en el aula una dinámica activa, en la que se demanda una participación mayor del profesor que la dada en clase tradicional.

No es trivial por tanto que los estudiantes reafirmen la figura del profesor como esencial en la dinámica escolar, lo que confirma que el uso de dicha tecnología es vista como mediadora del aprendizaje, y no un elemento que "desplaza" al profesor de su rol. Este hallazgo se constituye en un indicador de observación relevante en las experiencias piloto que se están desarrollando actualmente en los diferentes centros escolares. Asimismo, se configura en una línea de aplicación práctica, donde se hace necesario la capacitación a los profesores en la incorporación a su bagaje pedagógico de las estrategias que le permitan alcanzar este nuevo rol y así poder desempeñarse como actores del proceso de enseñanza aprendizaje en forma eficiente.

Respecto del impacto que tiene la herramienta en los logros que obtienen los estudiantes, los resultados de este estudio muestran cómo los estudiantes y el profesor reportan más beneficios sociales, como el trabajo en equipo para resolver la tarea, la disposición para escuchar y ayudar al otro, que logros académicos en el área. Cuánto saben y qué es lo que saben, son preguntas que aunque no son objeto de investigación de este estudio, el profesor como los estudiantes consideran que no han aprendido más de lo que ocurre en una clase tradicional aunque reportan más aprendizajes en relación al razonamiento y planificación previa al resolver un problema.

Los hallazgos revelan que los criterios usados para la organización de los grupos son elementos centrales que definen el clima generado al trabajar con ADP al interior del aula. Haber ensayado la organización al azar en esta muestra de estudiantes, nos permitió identificar que es necesario el estudio de otras direcciones en este aspecto para refinar la manera en que se organizan los grupos. La apreciación de los estudiantes de organizarlos por niveles de desempeño es una alternativa que se corresponde igualmente con la teoría que sustenta este tipo de aprendizaje (Dillenbourg, 1999), y que puede considerarse en futuras aplicaciones con el fin de generar modelos de organización cada vez más completos que permitan un equilibrio al interior del grupo.

Dicho estudio mostró que los roles que desempeñan los estudiantes dentro del grupo dependen de cómo estén organizados. Por ejemplo, los estudiantes estaban dispuestos a participar activamente y a generar explicaciones para resolver la tarea si se trabajaba en equipo. Es decir, si existe la idea de que trabajar colaborativamente les permite alcanzar el objetivo. Dicha condición está relacionada con la claridad que se tenga de los roles a desempeñar al interior de un grupo, de lo contrario es difícil trabajar colaborativamente pues surgen conductas disruptivas que imposibilitan el trabajo. La poca claridad por parte de algunos estudiantes de cómo interactuar dentro del grupo, imposibilitó en ellos su avance en la dinámica así como no poder identificar-más allá de los resultados obtenidos en relación a los tipos de intervenciones- cuáles son los roles fundamentales que se encuentran a la base de las interacciones. En base a lo anterior, se confirma que el uso de esta herramienta tiene implicancias importantes en el estudio de las interacciones que se generan en esta dinámica y que por tanto, su estudio es relevante a nivel práctico y teórico. Éste se constituye en un aspecto de interés que debe profundizarse en un tiempo y población mayor, del utilizado para este estudio.

En dichos hallazgos, se entremezclan elementos del campo psicológico, que este estudio reafirma, a saber: (a) algunas herramientas cognitivas potencian o impulsan el desarrollo de habilidades en los estudiantes -en este caso, habilidades de razonamiento y planificación-, (b) el trabajo en conjunto con pares permite la construcción de conocimiento en conjunto gracias a la posibilidad de compartir sus rutas de razonamiento o simplemente ideas tentativas de solución a la tarea, (c) el profesor se constituye en un facilitador del proceso de aprendizaje en sus estudiantes; un elemento clave en el andamiaje de conocimientos en los estudiantes, (d) la forma como se estructura el contexto favorece el desarrollo de determinadas habilidades sociales y/o cognitivas en los estudiantes gracias a la interrelación de las acciones sociales o intervenciones y las actividades producidas al interior de los grupos.

En relación al campo tecnológico dicho estudio nos provee rica información sobre la forma como podría estar estructurada la herramienta para que 
fuese más provechosa en el campo educativo. Por un lado, los estudiantes afirman que un sistema más flexible, dinámico y con incentivos podría constituirse en una herramienta que los motivara mucho más a apropiarse de su aprendizaje y del desarrollo de la clase. Por otro, consideran que una estructura que les proporcione información del por qué está incorrecto el razonamiento más que la respuesta, así como la posibilidad de integrar al trabajo estudiantes universitarios como facilitadores de este proceso, son elementos complementarios al sistema.

Las consideraciones que apuntan a la estructura del sistema son primordiales en el campo de la evaluación instruccional pues justamente permiten un enriquecimiento del sistema y un acercamiento más real al contexto escolar. En este sentido, es relevante destacar cómo estos aportes han sido contemplados como futuros desarrollos para el sistema, por parte del equipo que trabaja en el proyecto Fondef (Rosas et al., 2003). Las consideraciones que guardan relación con la posibilidad de interactuar en este proceso con estudiantes universitarios como método de salvar las deficiencias en la atención que debe brindar el profesor a cada grupo, es una idea interesante de estudiar y de ver si efectivamente puede implementarse y, más allá de esto, reporta beneficios tanto a la dinámica misma como al aprendizaje de los estudiantes.

Desde nuestra mirada, el uso de ADP propicia tanto en estudiantes como en profesores un desempeño activo y en conjunto para resolver la tarea y genera tipos de interacciones necesarias de estudiar en especial si favorecen el proceso de enseñanza-aprendizaje. Los estudiantes reconocen que para poder avanzar en la tarea deben explicar a otros cuando no entienden, recibir ayuda para entender, construir en conjunto una solución para obtener un resultado. De la misma manera, permitió evidenciar que desde la mirada de los estudiantes el profesor es una pieza clave en la dinámica escolar que se genera con el uso de ADP. No menos importante, permitió evidenciar cómo el uso de la tecnología se convierte en un artefacto -siguiendo a Vigotsky- que media en el proceso de aprendizaje intergrupal e intraindividual de los estudiantes, así como también los procesos sociales dados en el aula.

\section{Referencias}

Barbieri, M. S. \& Light, P. H. (1992). Interaction, gender, and performance in a computer-based problem solving task. Learning and Instruction, 2, 191-213.

Barros, B., Mizoguchi, R. \& Verdejo, M. (2001). A platform for collaboration analysis in CSCL. An ontological approach. En Proceedings of the Artificial Intelligence in Education AIIED'2001. Recuperado el 20 de octubre de 2004, desde http://sensei.ieec.uned.es

Chiu, Ch. (2003). Exploring how primary school student's function in computer supported collaborative learning. International Journal of Continuing Engineering Education and Life Long Learning, 13(3, 4), 258-267.

Danesh, A., Inkpen, K., Lau, F., Shu, K. \& Booth, K. (2001). Geney TM: Designing a collaborative activity for the palm TM handheld computer. Recuperado el 25 de abril del 2003, desde http//www.edgelab.sfu.ca/publications/ chi2001_geney.pdf

Dillenbourg, P. (1999). Collaborative learning. Cognitive and computational approaches. New York: Pergamon Earli.

Hakkarainen, K. \& Palonen, T. (2003). Patterns of female and male students' participation in peer interaction in computer-supported learning. Computers \& Education, 40(4), 327-342.

Inaba, A., Tamura, T., Ohkubo, R., Ikeda, M., Mizoguchi, R. \& Toyoda, J. (2001). Design and analysis of learners' interaction based on collaborative learning ontology. En Proceedings of Euro-CSCL2001 (pp. 308-315). Recuperado el 27 de octubre de 2004, desde http:// www.ei.sanken.osaka-u.ac.jp/ ina/Doc/Ref/L3.htm

Gifford, B. \& Enyedy, N. (1999). Activity centered design: Towards a theoretical framework for CSCL. En Proceedings of the Third International Conference on Computer Support for Collaborative Learning. Recuperado el 27 de octubre de 2004, desde www.gseis.ucla.edu/ faculty/enyedy/pubs/ Gifford\&EnyedyCSCL2000.pdf

Gillies, R. M. (2003). The behaviors, interactions, and perceptions of junior high school students during smallgroup learning. Journal of Educational Psychology, 95(1), 137-147.

Jones, C., Connolly, M., Gear, A. \& Read, M. (2001). Group interactive learning with group process support technology. British Journal of Educational Technology, 32(5), 571-586.

Kynigos, C. (1999). Perspectives in analyzing classroom interaction data on collaborative computer-based mathematical projects. En C. Hoadley \& J. Roschelle (Eds.), Proceedings of the Computer Support for Collaborative Learning (CSCL) 1999. Mahwah, NJ: Lawrence Erlbaum Associates.

Lea, M., Rogers, P. \& Postmes, T. (2002). SIDE-VIEW: Evaluation of a system to develop team players and improve productivity in Internet collaborative learning groups. British Journal of Educational Technology, 33(1), 53-63.

Minick, N. (1997). The early history of the Vigotskian school: The relationship between mind and activity. En M. Cole, Y. Engeström \& O. Vasquez (Eds.), Mind, culture and activity. Seminal papers from the laboratory of comparative human cognition (pp. 117-127). New York: Cambridge University Press.

Pownell, D. \& Bailey, G. (2000). Handheld computing for educational leaders: A tool for organizing or empowerment. Leading \& Learning with Technology, 27(8), 46-49, 5960.

Preiss, D. \& Sternberg, R. J. (2006). Effects of technology on verbal and visual-spatial abilities. International Journal of Cognitive Technology, 11(1), 14-22.

Rosas, R., Nussbaum, M., Marianov, V., Correa, M., Flores, P., Grau, V. et al. (2003). Diseño y desarrollo de un siste- 
ma generador de pruebas de evaluación de aprendizaje escolar a través de computadores portátiles inalámbricos. Revista Pensamiento Educativo, 33, 128-159.

Roschelle, J. \& Pea, R. (2002). A walk on the WILD side: How wireless handhelds may change computer supported collaborative learning. Recuperado el 20 de octubre de 2004, desde http://newmedia.colorado.edu/cscl/ 79.pdf

Roschelle, J., Pea, R., Hoadley, C., Gordin, D. \& Means, B. (2001). Changing how and what children learn in school with computer-based technologies. The future of children, 10(2), 76-101.

Salomon, G. (1993). Distributed cognitions: Psychological and educational considerations. New York, NY: Cambridge University Press.

Schaumburg, H. (2001). The impact of mobile computers in the classroom. Results from an going video study. Recuperado el 27 de octubre de 2005, desde http:// usight.concord.org/documents/electronic_guidebook.pdf.

Tatar, D., Roschelle, J., Vahey, P. \& Penuel, W. (2003). Handhelds go to school: Lessons learned. Published by the IEEE Computer Society.

Vahey, P. \& Crawford, V. (2003). Learning with handhelds: Findings from classroom research. Recuperado el 8 de junio del 2003, desde http://www.intel.com/education/ handhelds/SRI.pdf

Fecha de recepción: Noviembre de 2005.

Fecha de aceptación: Marzo de 2007.
Veerman, A. (2000). Collaborative learning through electronic discussion. Recuperado el 20 de octubre de 2005, desde http://collide.informatik.uni-duisburg.de/ecai-2000/ W26 Veerman.pdf

Vigostky, L. (1978). Mind in society: The development of higher psychological process. Cambridge, MA: Harvard University Press.

Yarnall, L., Penuel, W., Ravitz, J., Murray, G. \& Means, B. (2001). Portable assessment authoring: Using handheld technology to assess collaborative inquiry. Paper to be presented at Annual Meeting of the American Educational Research Association, Seattle, Washington.

Zurita, G. \& Nussbaum, M. (2001). Mobile CSCL applications supported by mobile computing. AI-ED 2001. MultiAgent Architectures for Distributed Learning Enviroments Workshop, San Antonio, Texas, EEUU.

Zurita, G. \& Nussbaum, M. (2004). A constructivist mobile learning environment supported by a wireless handheld network. Journal of Computer Assisted Learning, 20, 235243.

Zurita, G., Nussbaum, M. \& Sharples, M. (2003). Encouraging face-to-face collaborative learning through the use of handheld computers in the classroom. En Mobile HCI 2003, Fifth International Symposium on Human Computer Interaction with Mobile Devices and Services (Vol. 2795, pp. 193-208). Udine, Italy. 